\title{
Quality Assurance in Biobanking for Pre-Clinical Research
}

\author{
Daniel Simeon-Dubach ${ }^{a} \quad$ Steffen M. Zeisberger ${ }^{b}$ Simon P. Hoerstrup ${ }^{c}$ \\ ${ }^{\text {a }}$ Medservice, Biobanking Consulting \& Services, Walchwil, Switzerland; \\ bWyss Translational Center Zurich, Regenerative Medicine Technologies Platform, University of Zurich and ETH Zurich, Zurich, Switzerland; \\ ${ }^{c}$ Wyss Translational Center Zurich, University of Zurich and ETH Zurich, and Institute of Regenerative Medicine, University of Zurich, \\ Zurich, Switzerland
}

\section{Keywords}

Translational medicine - Regenerative medicine . Biobank · Pre-clinical research · Clinical research · QA

\section{Summary}

It is estimated that not less than USD 28 billion are spent each year in the USA alone on irreproducible pre-clinical research, which is not only a fundamental loss of investment and resources but also a strong inhibitor of efficiency for upstream processes regarding the translation towards clinical applications and therapies. The issues and cost of irreproducibility has mainly been published on pre-clinical research. In contrast to pre-clinical research, test material is often being transferred into humans in clinical research. To protect treated human subjects and guarantee a defined quality standard in the field of clinical research, the manufacturing and processing infrastructures have to strictly follow and adhere to certain (inter-)national quality standards. It is assumed and suggested by the authors that by an implementation of certain quality standards within the area of pre-clinical research, billions of USD might be saved and the translation phase of promising pre-clinical results towards clinical applications may substantially be improved. In this review, we discuss how an implementation of a quality assurance (QA) management system might positively improve sample quality and sustainability within preclinically focused biobank infrastructures. Biobanks are frequently positioned at the very beginning of the bio-

Daniel Simeon-Dubach and Steffen M. Zeisberger contributed equally to this article. medical research value chain, and, since almost every research material has been stored in a biobank during the investigated life cycle, biobanking seems to be of substantial importance from this perspective. The role model of a QA-regulated biobank structure can be found in biobanks within the context of clinical research organizations such as in regenerative medicine clusters.

(c) 2016 S. Karger GmbH, Freiburg

\section{Introduction}

Next to numerous biomedical disciplines, regenerative medicine (RM) is one of the areas in academia and biomedical research where basic science, pre-clinical, translational, and clinical research as well as commercial manufacturing are tightly combined. Consequently, RM will be used as an example discipline, demonstrating the translational process from a less regulated towards a strictly regulated research organization with a focus on biobanking. The core of many RM centers is on one hand a laboratory where all preclinical research is conducted mainly without any quality assurance (QA) management and on the other hand a good manufacturing practice (GMP) production site where clinical-grade cellular, acellular, or biomaterial products are manufactured with high adherence to (inter-)national quality guidelines. One integral part of such an infrastructure is a biobank for collection, processing, and preservation of cells, tissues and products thereof for pre-clinical and clinical research purposes.

In this review, we will discuss how an implementation of a QA management system might improve sample quality and sustainability within pre-clinically focused biobanking infrastructures. The

\section{KARGER}

() 2016 S. Karger GmbH, Freiburg

Fax +497614520714 
role model of a QA-regulated biobank structure can be found in biobanks within the context of clinical research organizations such as in RM clusters.

As a basis, the terms 'translational medicine', 'regenerative medicine', and 'biobank / biobanking' need to be defined in order to be able to share a common language.

\section{Translational Medicine}

The definition of translational medicine or translational research is still under debate. The National Institute of Health (NIH) roadmap includes two major research domains (bench and bedside) and two 'translational' steps (T1 and T2) [1]. In translational step T1, basic science (pre-clinical research and animal studies) is translated into an understanding of basic human biochemistry and (patho-)physiology following mainly no QA regulations. In translational step T2, this knowledge is further translated into improved diagnostic tests, medicines, and treatments for use in clinical routine which is performed under (inter-)national regulations implemented and maintained by a QA management system. It is obvious that moving new discovery into clinical practice is challenging and might take a long time. It occurs largely through continuing medical education programs and guideline development. Consequently, in order to improve incorporation of research discoveries into dayto-day clinical care, an additional translational step (T3) was proposed [1] (fig. 1).

\section{Regenerative Medicine}

RM replaces or regenerates human cells, tissue, or organs to restore or establish normal function [2,3]. Since the ultimate aim of $\mathrm{RM}$ is to return the patient to full health with respect to the particular condition, 'repair' would fall within earlier technologies such as surgery. The central focus of RM is on human cells. These may be somatic, adult stem or embryo-derived cells, and today there are versions of the latter cells that have been reprogrammed from adult cells and both are grouped as 'pluripotent cells' [3]. RM is a broad field that includes tissue engineering but also incorporates research on self-healing - where the body uses its own systems, sometimes with help of foreign biological material, to recreate cells and rebuild tissues and organs [4]. At least in the academic settings, RM is often organized as a center of competence with close links to other areas within a health care center. An example of such a center of competence is the Mayo Clinic Center for Regenerative Medicine [5]. This center is divided in multiple subunits, e.g. for patient access, or in departments where translational research is conducted to improve existing applications or to develop new applications of RM. The core of such a translational center in $\mathrm{RM}$ is a laboratory where all the pre-clinical research is being conducted and a GMP production unit where the clinical-grade products are manufactured following (inter-)national regulations under a strict QA system.

\section{Biobank and Biobanking}

There is still not a commonly accepted definition of a biobank. Based on a survey amongst peers, the following definition for a biobank was proposed: 'A biobank is a facility for the collection, preservation, storage, and supply of biological samples and associated data, which follows standardized operating procedures and provides material for scientific and clinical use' [6]. This definition mirrors very well the one issued by the Organization for Economic Co-Operation and Development (OECD) some years ago [7]. An alternative way to describe and classify a biobank is by the numbers of potential users it is serving, ranging from mono-user to oligoand multi-users biobanks [8]. The majority of biobanks are monouser biobanks. Often these mono-user biobanks have no or only a minimal QA management in place. Especially in the academic setting, these mono-user biobanks and to a lesser extent the oligoand multi-user biobanks exist to support the research activities of one or a group of individuals, [9]. Both definitions mentioned above clearly imply that a biobank is an infrastructure with the primary aim to serve the research community.

The term 'biobanking' describes the process from the first attempt to contact the patient or donor to the moment when the samples and data have been transferred to be used for research and/or restocking of unused samples [10]. Biobanking is indispensable for determining the most effective way to store biospecimens and data and to make them available when required.

\section{Implementation of Quality Assurance in the Field of Clinical Research Might Have a Quality-Improving Impact on the Organization of a Biobank}

In clinical research, often test material will be transferred into humans. To protect treated human subjects and guarantee a defined quality standard, these trials have to follow good clinical practice (GCP) guidelines. If medicinal products are manufactured, depending on the individual regulations, also GMP standards or standards of the International Organization for Standardization (ISO) have to be implemented and adhered to. These quality standards guarantee clearly defined processes and quality control and have organizational and structural consequences within the processing unit. This implements initial and periodic qualifications of the tools and equipment. This process normally includes repetitive calibration of e.g. all temperature sensors used in the biobanking facility as well as a strict documentation of these activities. Installation, operation, and performance qualification of the installed infrastructure is an integral part of the QA system and required by (inter-)national quality guidelines guaranteeing consistency and sustainability of stored material and metadata. Further, an internal audit process as well as supplier QA is an integral part of the QA management to guarantee and maintain a certain quality standard. In contrast to biobanks of clinical trial organizations, currently only a small portion of pre-clinically focused biobanks are audited or inspected by external authorities in order to keep their opera- 
Fig. 1. The current National Institutes of Health (NIH) roadmap for medical research includes two major research laboratories (bench and bedside) and two translational steps (T1 and T2). Historically, moving new medical discoveries into clinical practice (T2) has been haphazard, occurring largely through continuing medical education programs, pharmaceutical detailing, and guideline development. Proposed expansion of the NIH roadmap (lower part) includes an additional research laboratory and translational step (T3) to improve incorporation of research discoveries into day-to-day clinical care. The research roadmap is a continuum, with overlap between sites of research and translational steps. The figure includes examples of the types of research common in each research laboratory and translational step. This map is not exhaustive; other important types of research that might be included are community-based participatory research, public health research, and healthy policy analysis (adapted from [1]).

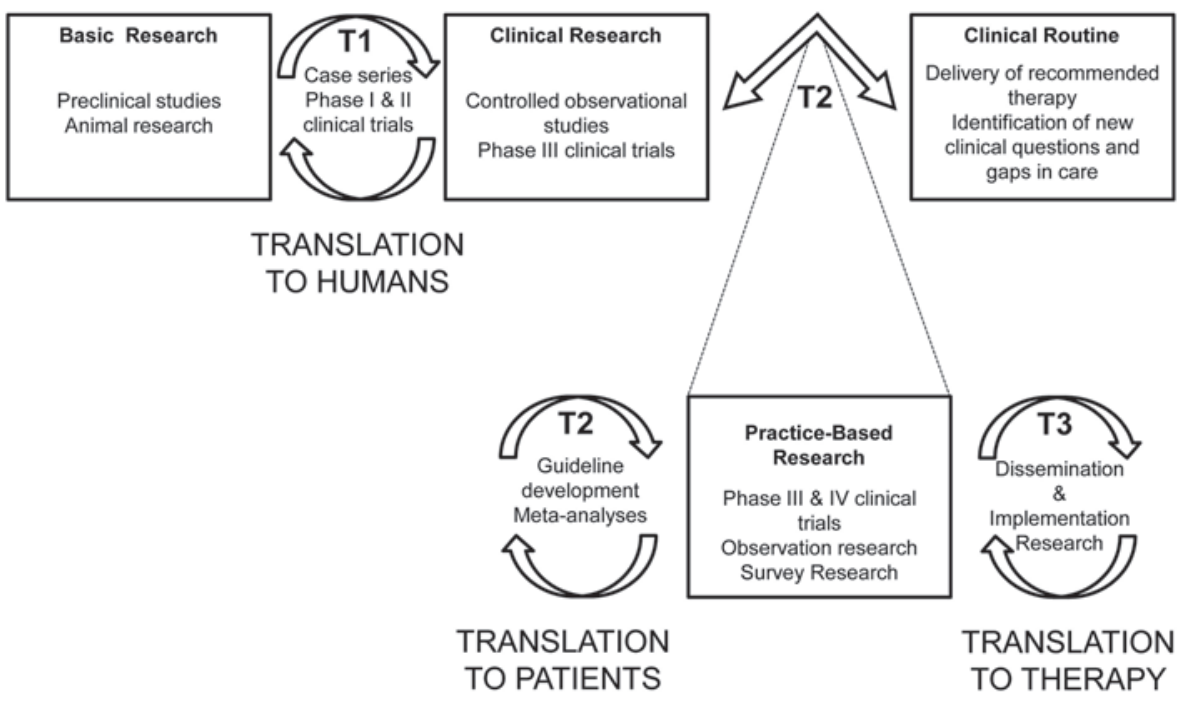

tions compliant to certain quality standards or current (inter-)national regulations. For clinical research organizations multiple (inter-)national registration bodies are in charge to enforce that all activities are compliant with the current quality regulations. The EU Regulation on Advanced Therapy Medicinal Products (known as the EU-ATMP Regulation) has been in force since 2008 and represents a significant step forward in the regulation of RM-based therapeutic products in general and in the regulation of tissue-engineered products in particular [11]. Comparable requirements for pre-clinical research activities are mostly missing. The question arises, why should evidence-based pre-clinical research not be conducted at a comparable or at least above a certain quality standard compared to clinical research and manufacturing organizations? It should be obvious that evidence-based pre-clinical research should be approached only under a certain QA standard, although it requires initially and sustained substantial investment in personnel and infrastructure resources. However, an up-to-date QA in biobanking infrastructure is costly and a major challenge for every biobank. Probably, centralization, e.g. of different biobanking research clusters towards a centrally guided QA management system, might help reducing the individual extra investments of a QA system, since the investment would need to be done only once instead.

\section{The Benefit of Introducing Quality Assurance for Pre-Clinical Research-Focused Biobanks}

Recent publications have focused on the increasing disparities between resource inputs and outcomes in biomedical research [12]. It was estimated that more than USD 28 billion are spent each year in the USA alone on irreproducible pre-clinical research, which is a fundamental loss for upstream processes regarding the translation towards clinical applications [13]. The issues and cost of irreproducibility has been published mainly on pre-clinical re- search [13-15]. Human biospecimens are used in approximately $40 \%$ of all research findings published in the biomedical literature [16], the vast majority of them used in pre-clinical research and sourced from pathology archives and from established biobanks [17]. All the mentioned issues have not been without any response of the research and biobanking community. In order to improve quality and reproducibility, many guidelines have been developed and introduced including a specific one for biobanking purposes, the so-called Biospecimen Reporting for Improved Study Quality (BRISQ) $[18,19]$. However, even after having been published simultaneously in several journals, BRISQ is still not broadly implemented in the most relevant journals.

Within the biobanking community, these issues are intensively discussed as biobanking becomes more professionalized one might see some move from a focus on high number of samples as first priority towards a main attention on quality of the samples [20]. A new research field called biospecimen research has been evolved, focusing on pre-analytical variables of samples. Extensive publications are available on this subject. In contrast, almost no papers are available describing process validations and qualifications of equipment used in biobanks, underlining the need for consistency and reproducibility. One early example comes from the UK Biobank where manufacturing standards to analyze sample processing have been used [21]. In contrast, only one single abstract describing freezer maintenance requirement based on periodic performance qualification have been found [22]. According to our knowledge, there are no publications comparing pre-clinical research conducted in a regulated versus that in an unregulated setting. One might speculate which impact the (non-)qualification of tools and equipment used in pre-clinical research activities might have. However, some countries like France have recently introduced stricter country-specific regulations for biobanking [23]. The ISO offers a range of broad standards that can be applied to biorepositories, for example, ISO 9001:2015 Quality Management Systems - Requirements [24]. ISO 9001:2015 has seven major sec- 
tions which include the context of the organization, leadership, planning, support, operations, performance evaluation, and improvement. Biorepositories that perform sample testing within medical laboratory workflows may be accredited to ISO 15189 Medical Laboratories - Requirements for Quality and Competence [25]. Another common standard is ISO/IEC 17025:200529, which specifies the general requirements for the competence to carry out tests and/or calibrations, including sampling [26]. It covers testing and calibration performed using standard methods, non-standard methods, and laboratory-developed methods and is applicable to all organizations performing tests and/or calibrations. In addition a technical committee of the ISO is about to establish quality standards specific for biobanks $[27,28]$. Its release is expected for 2017/2018. The working group specific to biobanking, WG2: Biobanks and Bioresources, took the approach of drawing from existing best practice documents in order to identify the key foundational principles [29]. As of today two comprehensive QA programs for biobanking are available: the Canadian Tissue Repository Network (CTRNet) Biobank Certification Program and the College of American Pathologist (CAP) accreditation program. There are also published methodologies and other tools to support biobanking quality. These include the use of intra-biorepository methods [30-33] and inter-biorepository comparisons to determine performance using specific tests or measurements [34-37] and the Self-Assessment Tool (SAT) and the Pre-Analytical External Quality Assurance (EQA) Survey. Both SAT and EQA are provided by the International Society of Biological and Environmental Repository (ISBER) [38].

Many countries will adapt these standards. Some individual biobanks might not be able to follow sufficiently these standards and might merge with others with the aim conducting a shared QA-based sample management [39, 40]. QA standards might most probably even more efficiently established and maintained in a centralized infrastructure, because of a higher degree of similar workflows, environmental and infrastructural settings and parameters. Ultimately, biobanks need to define the potential end-user prospectively very early in the collecting process. The collection process should then further move from a quantity and quality focus to a customer focus, i.e., biobanking 3.0 [20]. It has been shown that staying in tune with the customers of the research community will become critical to survive [41]. Professionalization of biobanking including a detailed business plan will also be very helpful [42]. Financing and return of investment will be a major issue, and public money will be needed as for any other infrastructure that serves a larger community. Biobanks using QA-controlled workflows and equipment will be able to provide samples and data of clearly defined quality to the research community. At the end, this probably might help to reduce the billions of USD that are used annually in irreproducible pre-clinical research [13].

\section{Acknowledgments}

SPH and SMZ are grateful to the Maexi-Foundation and the Center for Applied Biotechnology and Molecular Medicine (CABMM) for financial support.

\section{Disclosure Statement}

The authors have no conflict of interest.

\section{References}

1 Westfall JM, Mold J, Fagnan L: Practice-based research - 'Blue Highways' on the NIH roadmap. JAMA 2007; 297:403-406.

2 BSI: PAS 84:2012 Cell therapy and regenerative medicine. Glossary. http://shop.bsigroup com/Browse-by-Sector/ Healthcare/PAS-84/ (last accessed August 23, 2016).

3 Mason C, Dunnill P: A brief definition of regenerative medicine. Regen Med 2008;3:1-5

4 National Institute of Biomedical Imaging and Bioengineering: Tissue Engineering and Regenerative Medicine. http://www nibib.nih.gov/science-education/sciencetopics/tissue-engineering-and-regenerative-medicine (last accessed August 23, 2016)

5 Terzic A, Pfenning MA, Gores GJ, Harper CM Jr: regenerative medicine build-out. Stem Cells Transl Med 2015;4:1373-1379

6 Hewitt R, Watson PH: Defining biobank. Biopreserv Biobank 2013;11:309-315.

7 OECD: Creation and Governance of Human Genetic Databases. 2006. www.oecd.org/sti/biotech/creationandgovernanceofhumangeneticresearchdatabases.htm (last accessed August 23, 2106)

8 Watson PH, Barnes RO: A proposed schema for classifying human research biobanks. Biopreserv Biobank 2011;9:327-333.
Yuille M: Brave New World. Biopreserv Biobank 2015; 13:433-434.

10 Vaught J, Rogers J, Myers K, Lim MD, Lockhart N, Moore H, Sawyer S, Furman JL, Compton C: An NCI perspective on creating sustainable biospecimen resources. J Natl Cancer Inst Monogr 2011;42:1-7.

11 Brevignon-Dodin L: Regulatory enablers and regulatory challenges for the development of tissue-engineered products in the EU. Biomed Mater Eng 2010;20:121126.

12 Bowen A, Casadevall A: Increasing disparities between resource inputs and outcomes, as measured by certain health deliverables, in biomedical research. Proc Natl Acad Sci U S A 2015;112:11335-11340.

13 Freedman LP, Cockburn IM, Simcoe TS: The economics of reproducibility in preclinical research. PLoS Biol 2015;13:e1002165

14 Begley CG, Ellis LM: Drug development: raise stand ards for preclinical cancer research. Nature 2012;483: 531-533.

15 Prinz F, Schlange T, Asadullah K: Believe it or not: how much can we rely on published data on potential drug targets? Nat Rev Drug Discov 2011;10:712.

16 Hughes SE, Barnes RO, Watson P: Biospecimen use in cancer research over two decades. Biopres Biobank 2010; 8:89-97.
7 Castillo-Pelayo T, Babinszky S, LeBlanc J, Watson PH: The importance of biobanking in cancer research. Biopreserv Biobank 2015;13:172-177.

18 Simera I, Moher D, Hirst A, Hoey J, Schulz KF, Altman DG: Transparent and accurate reporting increases reliability, utility, and impact of your research: reporting guidelines and the EQUATOR Network. BMC Med 2010;8:24.

19 Moore HM, Kelly AB, Jewell SD, McShane LM, Clark DP, Greenspan R, Hainaut P, Hayes DF, Kim P, Mansfield EA, Potapova O, Riegman P, Rubinstein Y, Seijo E, Somiari S, Watson P, Weier HU, Zhu C, Vaught J: Biospecimen reporting for improved study quality (BRISQ). Biopreserv Biobank 2011;9:57-70.

20 Simeon-Dubach D, Watson P: Biobanking 3.0: evidence based and customer focused biobanking. Clin Biochem 2014;47:300-308.

21 Downey P, Peakman TC: Design and implementation of a high-throughput biological sample processing facility using modern manufacturing principles. Int $\mathrm{J}$ Epidemiol 2008;37(suppl 1):i46-i50.

22 Berman S, Wexelblat S, Pool T, Moore T, Shea K: Statistical methods for determining freezer maintenance requirements. Biopreserv Biobank 2012;10:216-QAC18. 
23 French National Standards: NF S 96-900 Certification of Biological Resource Centres. www.p3gobservatory. org/download/projet+norme+Biobanque+Eng pdf (last accessed August 23, 2016)

24 International Standard Organisation (ISO): ISO 9001: 2015 Quality Management Systems - Requirements. $w w w$.iso.org/iso/catalogue_detail?csnumber $=62085$ (last accessed August 23, 2016).

25 International Standard Organisation (ISO): ISO 15189: 2012 Medical Laboratories - Requirement for Quality and Competence. www.iso.org/iso/catalogue_detail? csnumber $=56115$ (last accessed August 23, 2016).

26 International Standard Organisation (ISO): ISO/IEC 17025:2005 General Requirements for the Competence of Testing and Calibration Laboratories. www.iso.org/ iso/catalogue_detail htm? csnumber $=39883$ (last accessed August 23, 2016).

27 International Standard Organisation (ISO): ISO/TC 276 Biotechnology. www.iso.org/iso/home/standards development/list_of_iso_technical_committees/iso_ technical_committee htm?commid $=4514241$ (last accessed August 23, 2016)

28 Furuta K, Schacter B: Report on Status of ISO276/ WG2 on biobanks and bioresources: international standards for biobanking. Biopreserv Biobank 2015;13: 452-453.

29 ISBER: 2012 best practices for repositories: collection, storage, retrieval, and distribution of biological material for research. Biopreserv Biobank 2012;10:79-161.

30 Kofanova OA, Mathieson W, Thomas GA, Betsou F: DNA fingerprinting: a quality control case study for human biospecimen authentication. Biopreserv Biobank 2014;12:151-153.
Betsou F: Biospecimen processing method validation. Biopreserv Biobank 2015;13:69.

32 Geddes TJ, Ketelsen BE, Ahmed S, Pruetz BL, Fortier LE: Utilizing in-house resources to correct sample mixups in a medium throughput biorepository: a case study. Biopreserv Biobank 2016;14:75-80.

33 Parrish RS, Garafalo AV, Ndifor V, Goetz KE, Reeves MJ, Yim A, Cooper RC, Iano-Fletcher J, Wang X, Tumminia SJ: Sample confirmation testing: a short tandem repeat-based quality assurance and quality control procedure for the eyeGENE biorepository. Biopreserv Biobank 2016;14:149-155.

34 Carithers LJ, Ardlie K, Barcus M, Branton PA, Britton A, Buia SA, Compton CC, DeLuca DS, Peter-Demchok J, Gelfand ET, Guan P, Korzeniewski GE, Lockhart NC, Rabiner CA, Rao AK, Robinson KL, Roche NV, Sawyer SJ, Segre AV, Shive CE, Smith AM, Sobin LH, Undale AH, Valentino KM, Vaught J, Young TR, Moore HM: A novel approach to high-quality postmortem tissue procurement: the GTEx project. Biopreserv Biobank 2015;13:311-319.

35 Le PC, Kobel M, de LM, Rahimi K, Madore J, Babinszky S, Bachvarov DR, Bachvarova M, Beauchamp MC, Cass CE, Chadwick D, Colleen C, Damaraju S, Dufour J, Gotlieb WH, Kalloger SE, Portelance L, McAlpine JN, Matte I, Piche A, Shaw P, Roehrl MH, Vanderhyden BC, Watson PH, Huntsman DG, Provencher DM, Mes-Masson AM: Specimen quality evaluation in Canadian biobanks participating in the COEUR repository. Biopreserv Biobank 2013; 11:83-93.
36 Ahmad-Nejad P, Duda A, Sucker A, Werner M, Bronsert P, Stickeler E, Reifenberger G, Malzkorn B, Oberlander M, Habermann JK, Bruch HP, Linnebacher M, Schadendorf D, Neumaier M: Assessing quality and functionality of DNA isolated from FFPE tissues through external quality assessment in tissue banks. Clin Chem Lab Med 2015;53:1927-1934.

37 Betsou F, Sobel ME: The ISBER biorepository proficiency testing program: two successful years already, and new features to come. Biopreserv Biobank 2013; 11:255-256.

38 ISBER: International Society of Biological and Environmental Repositories. www.isber.org (last accessed August 23, 2016)..

39 Stephens N, Dimond R: Closure of a human tissue biobank: individual, institutional, and field expectations during cycles of promise and disappointment. New Genet Soc 2015;34:417-436.

40 Baker TP, Mateczun JM, Rice CL: Military institute: US pathology centre units will live on. Nature 2011; 477:407.

41 Galbraith JW: Sustainability in the modern biorepository environment from the perspective of the tissue procurement core at the University of Iowa. Biopreserv Biobank 2015;13:430-432.

42 Uzarski D, Burke J, Turner B, Vroom J, Short N: A plan for academic biobank solvency-leveraging resources and applying business processes to improve sustainability. Clin Transl Sci 2015;8:553-557. 